\title{
ON THE SEMI-SUB-HYPERGROUPS OF A HYPERGROUP
}

\author{
CH. G. MASSOUROS \\ 54 Kllous Street \\ 15561 Cholargos \\ Athens, Greece \\ (Received October 20, 1987 and in revised form December 5, 1989)
}

\begin{abstract}
In this paper we study some properties of the semi-sub-hypergroups and the closed sub-hypergroups of the hypergroups. We introduce the correlated elements and the fundamental elements and we connect the concept antipodal of the latter with Frattin's hypergroup. We also present Helly's Theorem as a corollary of a more general Theorem.
\end{abstract}

KEY WORDS AND PHRASES. Hypercomposition, Hypergroup, Convex sets. 1980 AMS SUBJECT CLASSIFICATION CODE. 20 N99.

\section{INTRODUCTION.}

In 1934 F. Marty introduced a new mathematical structure which he called hypergroup (see Marty [1]). A hypergroup $(H,$.$) , is a non-vold set H$ endowed with a hypercomposition "." (i.e. a mapping of $H \times H$ into the set $P(H)$, of all subsets of $H$ ) which satisfies the following axioms:
1) $(x \cdot y) \cdot z=x \cdot(y \cdot z)$
for every $x, y, z \in H$ (associative axiom)
ii) $\mathrm{x} \cdot \mathrm{H}=\mathrm{H} \cdot \mathrm{x}=\mathrm{H}$
for every $x \in H$ (reproductive axiom)

It holds that $x \cdot y \neq \phi$ for every $x, y \in H$ (see Mittas [2]). We note that if "." is a hypercomposition in a set $H$ and $A, B$ are subsets of $H$, then A.B signifies the union $U(\alpha, b) \in A \times B$ a.b. $A \cdot b$ and $a \cdot B$ will have the same meaning as $A \cdot\{b\}$ and $\{a\} \cdot B$ respectively. We also make no distinction between the elements and their corresponding singletons, when nothing opposes it.

The hypercomposition "." leads, in a quite natural way, to two new hypercompositions ":" and ".." which are defined as follows (see also [1]):

$$
\begin{aligned}
& a: b=\{x \in H \mid a \varepsilon x \cdot b) \\
& a \cdot b b=\left\{\begin{array}{lll}
x & =H \mid a \varepsilon b \cdot x
\end{array} .\right.
\end{aligned}
$$


Whenever "." is conmutative, then $a: b=a . . b$ for $a l l a, b \varepsilon H$. Besides one can eas $t l y$ observe that $a: b \neq \phi, a . . b \neq \phi$. Indeed since $H=H \cdot b=b . H$ there is at least one $x$ and one $y$ such that $a \varepsilon x \cdot b$ and $a \varepsilon b . y$. Therefore, the sets $a: b$ and $a . . b$ are non void.

A subset $K$ of the hypergroup $\Omega$ will be called a semi-subhypergroup of $\Omega$ if $a \cdot b \subseteq K$ holds for every $a, b \varepsilon K$, while it is called a sub-hypergroup of $\Omega$, if the reproductive axiom holds in $K$ as well ( $1 . e$. $a \cdot K=K \cdot a=K$ for every $a \varepsilon K$ ). It is easy to prove that if in a semi-sub-hypergroup $K$, is valid $a: b \subseteq K$ and $a . b \subseteq K$, for $a 11 \mathrm{a}, \mathrm{b} \varepsilon \mathrm{K}$, then $\mathrm{K}$ is a sub-hypergroup of $\Omega$. Indeed, the proof of the regnerative axiom is as follows: Let $a \varepsilon K$. Then $a . K \subseteq K$ and $K . a \subseteq K$. Next, since r..a $\subseteq K$, $r: a \subseteq K$, for every $r \in K$, we have: $r \subseteq a \cdot K$ and $r \subseteq K . a$ respectively. Thus $K \subseteq$ a.K and $K \subseteq K \cdot a$, and so $K=K \cdot a=a \cdot K$.

A sub-hypergroup $K$ of $\Omega$ is called closed from the right (resp. from the left), if a.K $\cap K=\phi$ (resp. K.a $\cap K=\phi$ ) holds for every a $\varepsilon \Omega-K$. $K$ is called closed if it is closed from the righl and from the left. For the closed sub-hypergroups this proposition is valid: $K$ is closed from the right if and only if the relation a. $K \cap K \neq \phi$ implies that a $\varepsilon k$ (resp. from the left) (see also Mittas [2],[3] and Krasner [4]).

Undoubtedly the hypergroup can be considered as one of the most general structures of the abstract Algebra. Therefore, even the study of its elementary properties, imposes technical difficulties on the whole procedure.

The hypergroup, very often, is endowed with axioms which vary in strength and which make it a less general structure than the one that was initially introduced, and so, the research on this field is led through more concrete paths. So for instance F. Marty considered hypergroups, that have at least one bilaterally unit element and which he named regular hyergroups. He also considered regular hypergroups $\mathrm{H}$, such that for every element a $\varepsilon H$ there exists at least one $a^{\prime} \varepsilon H$ such that

e $\varepsilon a_{\cdot} a^{\prime} \cap a^{\prime} \cdot a$, where $e$ is a unit of $H$. He named these hypergroups completely regular hypergroups. A special form of a completely regular hypergroup is the canonical hypergroup. It has a unit element, which is scalar ( $s \in H$ is called scalar, if for every $x \in H$ the sets $s . x$ and $x . s$ are singletons) and a unique inverse for every of its elements (see Mittas [2], Roth [5]). The hypergroup has also been used as a basis for the creation of other algebraic structures, which were born out of a mathematical need (exampli gratia see Krasner [6]). Some examples of such structures are the hyperfields and the hyperrings (eg. Massouros [7], Nakassis [8]), the hypermodules, the vector hyperspaces (Massouros [9], Mittas [10]) etc.

Numerous papers based either on the research of the algebraic structure of hypergroups, or on the study and the development of the applications that can possibly derive from them, have been published since 1934. Today the research leads to some more specific hypergroups, but Marty's inftial hypergroup has not been fully investigated and there is the possibility for further research. In this paper we present a series of properties and we study some special categories of elements, and 
the correlated elements and the fundamental elements, and motivated by the latter we introduce Frattini's semi-sub-hypergroup. Also we prove a theorem, which, in our opinion, is the most general form that one can give in Helly's Theorem.

2. SOME PROPERTIES OF THE SEMI-SUB-HYPERGROUPS AND THE SUB-HYPERGROUPS.

Let us begin this section by giving some examples of hypergroups.

(i) Let $H$ be a non-void set with card $H>4$. We introduced in it a hypercomposition "." as follows:

We define an element $e$ to be the neutral element of $H$, that is $a \cdot e=e . a=a$ for every a $\varepsilon H$.

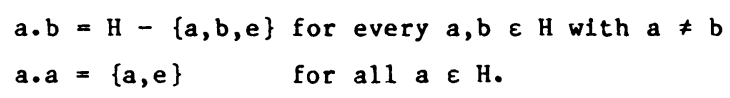

Then $(\mathrm{H},$. ) becomes a hypergroup (see also Nakassis [8]). For the two operations ":" and ".." we have:

$$
\begin{aligned}
& a: b=\{x \in H \mid a \varepsilon x \cdot b\}=H-\{a, b\}=b: a \\
& a: a=\{x \in H \mid a \in x \cdot a\}=\{a, e\}
\end{aligned}
$$

and because of the commutativity of ".", $a: b=a . . b$.

(ii) Let $\mathrm{H}$ be a non-void set and "." a hypercomposition defined as follows:

$a \cdot b=\{a, b\}$ for every $a, b \in H$ with $a \neq b$

a.a $=\mathrm{H} \quad$ for all a $\varepsilon \mathrm{H}$

Then $(\mathrm{H},$.$) is a hypergroup and for the two operations ":" and ".." we have:$

$a: b=\{x \in H \mid a \in x \cdot b\}=\{a, b\}=b: a$

$a: a=\left\{\begin{array}{lll}x & \varepsilon & H\end{array} \mid a \varepsilon x \cdot a\right\}=H$.

Because of the commutativity of "." we have: $a: b=a, . b$.

(iii) Let $H$ be a non-vold set. We introduce in $H$ a hypercomposition "." defined as follows:

$$
\begin{array}{ll}
a \cdot b=\{a, b\} & \text { for every } a, b \in H \text { with } a \neq b \\
a \cdot a=H-\{a\} & \text { for } a 11 a \varepsilon H .
\end{array}
$$

Then $(H,$.$) becomes a hypergroup (also see Massouros [11]) and for the two operations$ ":" and ".." we have:

$$
\begin{aligned}
& a: b=\left\{\begin{array}{lll}
x & \varepsilon & H
\end{array} \mid \begin{array}{lll}
a & \varepsilon & x \\
a
\end{array}\right\}=\{a, b\}=b: a \\
& a: a=\left\{\begin{array}{lll}
x & \varepsilon & H
\end{array} \mid \begin{array}{lll}
a & \varepsilon & x
\end{array}\right\}=H-\{a\} .
\end{aligned}
$$

Since "." is commutative $a: b=a . . b$ holds.

(iv) Let $\mathrm{H}$ be a group. We endow $\mathrm{H}$ with a hypercomposition "." defined as follows: $a \cdot b=\{a, b, a b\}$ for every $a, b \varepsilon H$.

Then $(\mathrm{H},$.$) becomes a hypergroup. In this hypergroup for the hypercompositions ":" and$ ".." we have:

$$
\begin{aligned}
& a: b=\left\{\begin{array}{lll}
x & \varepsilon & H
\end{array} \mid a \varepsilon x \cdot b\right\}=\left\{a, a b^{-1}\right\} \\
& a \cdot b=\left\{\begin{array}{lll}
x & \varepsilon & H
\end{array} \mid a \varepsilon b \cdot x\right\}=\left\{a, b^{-1} a\right\} .
\end{aligned}
$$

(v) Let $V$ be a vector space over an ordered field $F$. We introduce in $V$ a hypercomposition defiend as follows (also see Vougiouklis [12]):

$$
a \cdot b=\{\lambda a+k b \mid 0<\lambda, k: \lambda+k=1\} \text {. }
$$


Then (V.,) becomes a hypergroup. One can see that the convex sets of $V$ are simply the semi-sub-hypergroups of $(V,$.$) , while the subspaces of V$ are the closed sub-hypergroups of $(V,$.$) . So in some vector spaces, one can have an optical view of the$ hypercomposition, the semi-sub-hypergroups and the closed sub-hypergroups.

PROPOSITION 2.1. A sub-hypergroup $K$ of a hypergroup $\Omega$ is right closed (resp. left closed) if and only if $a: b \subseteq K$ (resp. $a . . b \subseteq K$ ) for every $a, b \in K$.

PROOF. Let us suppose that $K$ is a right closed hypergroup and let $a, b \in K$. Then for every $x \in a: b$ we have $a \varepsilon x \cdot b ;$ so $k \cap x \cdot k \neq \phi$. Thus, since $k$ is right closed, $x \in K$. Hence $a: b \subseteq K$. Conversely, let $K \cap x \cdot K \neq \phi$, for some $x \varepsilon \Omega$. Then there are $a, b \varepsilon K$ such that $a \varepsilon x \cdot b$, thus $x \varepsilon a: b$ and since $a: b \subseteq K$, we have $x \in K$.

PROPOSITION 2.2. Let $K$ be a closed sub-hypergroup of a hypergroup $\Omega$ and let

a $\varepsilon$ K. Then

$$
K=K: a=a: K=K . \cdot a=a . . K \text {. }
$$

PROOF. Since $K$ is a closed sub-hypergroup we have $K: a \subseteq K$. Now, let $x \in K$, then $x \cdot a \subseteq K$ and so $x \in K: a$. Thus $K \subseteq K: a$ and consequently $K=K: a$. Similarly the other equalities can be proved.

PROPOSITION 2.3. Let $(\mathrm{H},$.$) be a hypergroup. Then,$

i) $(a: b): c=a:(c \cdot b)$ and $(a, . b) \ldots c=a_{0} \cdot(b \cdot c)$

(ii) $b \in a \ldots(a: b)$ and $b \varepsilon a:(a \ldots b)$

PROOF. 1) Let $x \in(a: b): c$. Then $x \cdot c \cap a: b \neq \phi$, and therefore

$a \varepsilon(x \cdot c) \cdot b=x_{\cdot}(c \cdot b)$. So there exists an element $z \varepsilon c \cdot b$ such that $a \varepsilon x \cdot z$. Thus $x \in a: z$, and therefore $x \in a:(c \cdot b)$. Conversely, let $x \in a:(c \cdot b)$. Then

$a \varepsilon x \cdot(c \cdot b)=(x \cdot c) \cdot b$. Thus $x \cdot c \cap a: b \neq \phi$. Let $z \varepsilon x \cdot c \cap a: b$. Then $x \varepsilon z: c$ and therefore $x \in(a: b): c$. The proof of the other equality is analogous to this one.

(11) If $z \varepsilon a: b$, then, by definition, $a \varepsilon z \cdot b$. Therefore $a \varepsilon(a: b) \cdot b$. This means that there is an $x \in a: b$ such that $a \varepsilon x \cdot b$ or equivalently $b \varepsilon a \ldots x$. So $b \varepsilon a \ldots(a: b)$. Similarly one can prove that $b \varepsilon a:(a \ldots b)$.

SYMBOLISM. Let $E$ be a subset of a hypergroup $(H,$.$) Then [E], 〈E > will signify,$ the semi-sub-hypergroup and the closed sub-hypergroup of (H,.), respectively, which is generated from $E$ and contains 1t. Also, as a matter of, simplicity we shall write $\left[\alpha_{1}, \alpha_{2}, \ldots, \alpha_{n}\right]$ and $\left\langle\alpha_{1}, \alpha_{2}, \ldots, \alpha_{n}\right\rangle$ instead of $\left[\left\{\alpha_{1}, \alpha_{2}, \ldots, \alpha_{n}\right\}\right]$ and $\left\langle\left\{\alpha_{1}, \alpha_{2}, \ldots, \alpha_{n}\right\}\right\rangle$.

PROPOSITION 2.4. Let $\mathrm{H}, \mathrm{K}$ be two closed sub-hypergroups of a hypergroup $\Omega$, then

$$
\langle\mathrm{H} \quad \mathrm{K}\rangle=\langle\mathrm{H} \cdot \mathrm{K}\rangle=\langle\mathrm{H}: \mathrm{K}\rangle=\langle\mathrm{H} . \mathrm{K}\rangle\rangle
$$

SKETCH OF PROOF. Since H.K $\subseteq\langle\mathrm{HUK}\rangle$ we have that $\langle\mathrm{H} . \mathrm{K}\rangle \subseteq\langle\mathrm{HU} \mathrm{K}\rangle$. On the other hand $H \subseteq\langle H . K\rangle$ and $K \subseteq\langle H . K\rangle$. Indeed, for $H$, for Instance, we have:

$$
\begin{aligned}
\langle H . K\rangle \supseteq(H \cdot K):(H \cdot K) & =\text { (because of prop. 2.3,1) } \\
& =((H \cdot K): K): H \supseteq \\
& \supseteq H: H \approx \quad \text { (because of prop. 2.2) } \\
& =H
\end{aligned}
$$


Thus $\langle H \quad K\rangle \subseteq\langle H . K\rangle$ and so $\langle H U K\rangle=\langle H . K\rangle$. Analogous is the proof of the other equalities.

PROPOSITION 2.5. Let $A$ and $B$ be two subsets of a hypergroup $\Omega$ and $\mathrm{H}$ a closed subhypergroup of $\Omega$ such that $B \subseteq H$. Then:

i) $(A: B) \cap H=(A \cap H): B$

ii) $(A . . B) \cap H=(A \cap H) \ldots B$.

PROOF. From the relation $A \cap H \subseteq A$ we have

$$
(A \cap H): B \subseteq(A: B)
$$

Also from the relations $A \cap H \subseteq H$ and $B \subseteq H$ we have

$(A \cap H): B \subseteq H$

Thus, from the relations (2.1) and (2.2) we deduce that

$$
(A \cap H): B \subseteq(A: B) \cap H .
$$

Next let $x \in(A: B) \cap \mathrm{H}$.

Then there are $a \in A, b \in B$ such that $x \in a: b$ or equivalently a $\varepsilon$ x.b. But $x \cdot b \subseteq H$, thus a $\varepsilon H$ so a $\varepsilon A \cap H$. Therefore

$a: b \subseteq(A \cap H): B$ and thus $x \in(A \cap H): H$. So $(A: B) \cap H \subseteq(A \cap H): B$ and the proof is completed. With the same procedure we can prove (11).

PROPOSITION 2.6. Let A,B be two subsets of a hypergroup $\Omega$ and $\mathrm{H}$ a closed subhypergroup of $\Omega$ with $A \subseteq H$.

Then

$$
\text { (A.B) } \cap \mathrm{H}=\mathrm{A} \cdot(\mathrm{B} \cap \mathrm{H}) \text {. }
$$

PROOF. From the relation $A \cdot(B \cap H) \subseteq A . B, H$ we deduce that $A_{\cdot}(B \cap H) \subseteq(A . B) \cap H$. Next let $x \in(A . B) \cap \mathrm{H}$. Then there are $a \varepsilon A, \quad b \varepsilon$ B such that $x \varepsilon a \cdot b$, from where we have $b \varepsilon x \ldots a$ and $s 0 b \varepsilon H$. Thus $b \varepsilon B \cap H$ and therefore $x \in A \cdot(B \cap H)$. Hence (A.B) $\cap \mathrm{H} \subseteq \mathrm{A} .(\mathrm{B} \cap \mathrm{H}$ ) and so the proposition.

In Vouglouklis [12] p. 9 one can find interesting remarks on relations like the ones of propositions 2.6 and 2.7 .

PROPOSITION 2.7. Let $\mathrm{H}$ be a hypergroup and a $\varepsilon \mathrm{H}$.

Then

$$
[\alpha]=\alpha^{1} \cup \alpha^{2} \cup \ldots \cup \alpha^{K} \cup \ldots
$$

where $\alpha^{1}=\{\alpha\}, \alpha^{2}=\alpha \cdot \alpha$ and $\alpha^{1}=\alpha \cdot \alpha^{1-1}$.

SKETCH OF PROOF. Let $A$ be the right part of the above equality. Then it is obvious that $A \subseteq[\alpha]$. Next it is not difficult to show that $A$ is a semi-subhypergroup of $\mathrm{H}$ and moreover that it is the minimum one generated by $\alpha$ and containing it. 
REMARK 2.1 (i) If for some term of $A$, the relation $\alpha^{n} \subseteq \alpha^{1} \cup \alpha^{2} \cup \ldots \cup \alpha^{n-1}$ is valid, then we have that $\alpha^{n+r} \subseteq \alpha^{1} \cup \alpha^{2} U \ldots U \alpha^{n-1}$, for every $r \varepsilon N$ (see Vougiouklis [13]). (1) A semi-sub-hypergroup, which is generated by a single element is called a monogene (see Mittas [2]).

THEOREM 2.1. Let us consider a subset $E$ of a commutative hypergroup $H$. If $E=\left\{\alpha_{1}, \alpha_{2}, \ldots, \alpha_{n}\right\} \quad$ then

$$
\begin{aligned}
& {[E]=\left(\left[\alpha_{1}\right] \cup \ldots U\left[\alpha_{n}\right]\right) U} \\
& U\left(\left[\alpha_{1}\right] \cdot\left[\alpha_{2}\right] \cup \ldots U\left[\alpha_{n-1}\right] \cdot\left[\alpha_{n}\right]\right) U \\
& U . \ldots U \\
& U\left(\left[\alpha_{1}\right] \cdot \ldots \cdot\left[\alpha_{n}\right]\right)
\end{aligned}
$$

SKETCH OF PROOF. Let $A$ be the second part of the above equality. Since [E] is the semi-sub-hypergroup which is generated from $\alpha_{1}, \ldots, \alpha_{n}$ we have $\alpha_{1}, \ldots, \alpha_{n} \varepsilon[E]$ and one can easily see that [E] must contain all the sets of the form $\left[\alpha_{i}\right] \cdot \ldots \cdot\left[\alpha_{j}\right]$ with $1<i<j<n$. Thus $A \subseteq[E]$. Next, through not so difficult calculations, one can prove that $A$ is a semi-sub-hypergroup and moreover that it is the minimum one generated from [E].

PROPOSITION 2.8. In every commutative hypergroup $H$ the set

$\prod_{i=1}^{n}\left[\alpha_{1}\right]=\left[\alpha_{1}\right] \cdot\left[\alpha_{2}\right] \cdot \ldots \cdot\left[\alpha_{n}\right]$ is a semi-sub-hypergroup of $H$ that absorbs every $i=1$
element of $\left[\alpha_{1}, \alpha_{2}, \ldots, \alpha_{n}\right]$.

PROOF. In the beginning we observe that the set $\prod_{i=1}^{n}\left[\alpha_{1}\right]$ absorbs all the monogene semi-sub-hypergroups $\left[\alpha_{1}\right], 1<1<\mathrm{n}$. Indeed for every $\ell \varepsilon\{1,2, \ldots, \mathrm{n}\}$ we have

$$
\begin{aligned}
& \left(\left[\alpha_{1}\right] \cdot\left[\alpha_{2}\right] \cdot \ldots \cdot\left[\alpha_{n}\right]\right)\left[\alpha_{\ell}\right]=\left[\alpha_{1}\right] \cdot\left[\alpha_{2}\right], \underset{n}{\ldots} \cdot\left[\alpha_{n}\right] \cdot \\
& \text { From this relation we conclude that } \underset{i=1}{\prod}\left[\alpha_{1}\right] \text { absorbs every set of the form }
\end{aligned}
$$$$
\left[\alpha_{\ell 1}\right] . \ldots \cdot\left[\alpha_{\ell k}\right] \text { with } 1<\ell_{1}<\ldots<\ell_{k}<n \text {. Thus if } x, y \varepsilon \underset{1=1}{n}\left[\alpha_{1}\right] \text { then }
$$$$
x \cdot y \subseteq \prod_{i=1}^{n}\left[\alpha_{i}\right] \prod_{i=1}^{n}\left[\alpha_{i}\right]=\prod_{i=1}^{n}\left[\alpha_{i}\right] \text { and so } \prod_{i=1}^{n}\left[\alpha_{i}\right] \text { is a semi-sub-hypergroup of } H \text {. Next we }
$$

have

$$
\begin{aligned}
& \prod_{1=1}^{n}\left[\alpha_{1}\right] \cdot\left[\alpha_{1}, \alpha_{2}, \ldots, \alpha_{n}\right]= \\
& =\left(\left[\alpha_{1}\right] \cdot\left[\alpha_{2}\right] \cdot \ldots \cdot\left[\alpha_{n}\right]\right) . \\
& \text { - }\left(\left[\alpha_{1}\right] \cup \ldots \cup\left[\alpha_{n}\right] \cup\right. \\
& U\left[\alpha_{\ell 1}\right] \cdot\left[\alpha_{\ell 2}\right] \cdot \ldots \cdot\left[\alpha_{\ell k}\right] U \ldots \\
& \left.U\left[\alpha_{1}\right] \cdot\left[\alpha_{2}\right] \cdot \cdots \cdot\left[\alpha_{n}\right]\right)= \\
& =\left[\alpha_{1}\right] \cdot\left[\alpha_{2}\right] \cdot \ldots \cdot\left[\alpha_{n}\right] \cup \ldots \cup\left[\alpha_{1}\right] \cdot\left[\alpha_{2}\right] \cdot \ldots \cdot\left[\alpha_{n}\right]=\prod_{1=1}^{n}\left[\alpha_{1}\right] \cdot
\end{aligned}
$$


PROPOSITION 2.9. Let us consider a subset $E=\left\{\alpha_{1}, \alpha_{2}, \ldots, \alpha_{n}\right\}$, of a commutative hypergroup H. Then

$$
\left[\alpha_{1}, \alpha_{2}, \ldots, \alpha_{n}\right]=\left(\left[\alpha_{1}\right] \cup\left[\alpha_{2}\right] \cup \ldots \cup\left[\alpha_{n}\right]\right)^{n}
$$

PROOF. From the Theorem 2.1 we have

$$
\left[\alpha_{1}\right] \cup\left[\alpha_{2}\right] \cup \ldots \cup\left[\alpha_{n}\right] \subseteq\left[\alpha_{1}, \alpha_{2}, \ldots, \alpha_{n}\right]
$$

And since $\left[\alpha_{1}, \alpha_{2}, \ldots, \alpha_{n}\right]$ is a semi-sub-hypergroup, $\left(\left[\alpha_{1}\right] \cup\left[\alpha_{2}\right] \cup \ldots \cup\left[\alpha_{n}\right]\right)^{n}$ $\subseteq\left[\alpha_{1}, \alpha_{2}, \ldots, \alpha_{n}\right]$. Conversely. Let $x \in\left[\alpha_{1}, \alpha_{2}, \ldots, \alpha_{n}\right]$. Then there are $\alpha_{\ell 1}, \ldots, \alpha_{\ell k}$ with $1<\ell_{1}<\ldots<\ell_{K}<n$ such that $x \varepsilon\left[\alpha_{\ell 1}\right] . \ldots \cdot\left[\alpha_{\ell k}\right]$. In the case that $\ell_{k}<n$ we add to the above relation some more terms until we get $n$ terms.

$$
\left[\alpha_{\ell 1}\right] \cdot \ldots \cdot\left[\alpha_{\ell k}\right]=\left[\alpha_{\ell 1}\right] \cdot \ldots \cdot\left[\alpha_{\ell k}\right] \cdot \ldots \cdot\left[\alpha_{\ell k}\right]
$$

Thus we have

$$
\begin{gathered}
{\left[\alpha_{\ell 1}\right] \subseteq\left[\alpha_{1}\right] \cup \ldots \cup\left[\alpha_{n}\right]} \\
\vdots \\
{\left[\alpha_{\ell k}\right] \subseteq\left[\dot{\alpha}_{1}\right] \cup \ldots \cup\left[\alpha_{n}\right]} \\
\vdots \\
\cdot \\
{\left[\alpha_{\ell k}\right] \subseteq\left[\alpha_{1}\right] \cup \ldots \cup\left[\alpha_{n}\right] .}
\end{gathered}
$$

Therefore

$$
\left[\alpha_{\ell 1}\right] \cdot \ldots \cdot\left[\alpha_{\ell k}\right] \subseteq\left(\left[\alpha_{1}\right] \cup \ldots \cup\left[\alpha_{n}\right]\right)^{n}
$$

and the Proposition is proved.

\section{CORRELATED ELEMENTS.}

DEFINITION 3.1. In a hypergroup $H$ the elements $\alpha_{1}, \alpha_{2}, \ldots, \alpha_{n}$, will be called correlated if there are distinct integers $\lambda_{1}, \ldots, \lambda_{\nu}, \kappa_{1}, \ldots, k_{\mu}$ that belong to $\{1,2, \ldots n\}$ such that

$$
\left[\alpha_{\lambda 1}, \ldots, \alpha_{\lambda \nu}\right] \cap\left[\alpha_{K 1}, \ldots, \alpha_{K \mu}\right] \neq \phi \text {. }
$$

In the contrary case the elements $\alpha_{1}, \alpha_{2}, \ldots, \alpha_{n}$ will be called not correlated.

THEOREM 3.1. Let us suppose that the elements $\alpha_{1}, \ldots, \alpha_{n}$ of a hypergroup $\Omega$ are correlated. We consider all the semi-sub-hypergroups of $\Omega$ which are generated from n-1 elements of the above ones. Then the intersection of all these semi-subhypergroups is not void.

PROOF. Since the elements are correlated, there are distinct integers

$$
\begin{array}{r}
\kappa_{1}, \ldots, \kappa_{\nu}, \lambda_{1}, \ldots, \lambda_{\mu} \varepsilon\{1, \ldots, n\} \text { such that } \\
{\left[\alpha_{k 1}, \ldots, \alpha_{k \nu}\right] \cap\left[\alpha_{\lambda 1}, \ldots, \alpha_{\lambda \mu}\right] \neq \phi .}
\end{array}
$$


But every semi-sub-hypergroup which is generated by $n-1$ elements from the $\alpha_{1}, \ldots, \alpha_{n}$ contains one of the two semi-sub-hypergroups that are mentione in the above relation. So the elements of the intersection of the two sub-hypergroups of (3.1) belong to all the semi-sub-hypergroups that are produced from $n-1$ elements. Consequently these elements will also belong to the intersection of all these semisub-hypergroups and therefore thelr intersection will be non-void.

THEOREM 3.2. Let $\Omega$ be a hypergroup in whtch the elements of every set wilh cardinality greater than $n$, are correlated. Also let $\left(K_{i}\right)_{i \varepsilon I}$, with card $I>n$, be a finite family of semi-sub-hypergroups of $\Omega$. Then, if every $n$ of these semi-subhypergroups have non-void intersection, all of them have non-void intersection.

PROOF. We shall prove the theorem by induction. We shall first show that the intersection of every $n+1$ semi-sub-hypergroups is non-void. Without loss of generality we may prove this statement for the semi-sub-hypergroups $k_{i}, 1<i<n+1$. Thus every $t i m e$ we choose an element which belongs to the intersection of $n$ semi-subshypergroups of the $k_{i}, 1<1<n+1$. So let $x_{i} \in \cap_{j \neq 1} K_{j}$ Then

$$
x_{1}, \ldots, x_{i-1}, x_{1+1}, \ldots, x_{n+1} \varepsilon k_{i}
$$

and therefore

$$
\left[x_{1}, \ldots, x_{1-1}, x_{1+1}, \ldots, x_{n+1}\right] \subseteq K_{1} .
$$

But every $n+1$ elements of $\Omega$ are correlated, thus the elements $x_{1}, \ldots, x_{n+1}$ are correlated and because of the Theorem 3.1, the semi-sub-hypergroups

$$
\left.x_{1}, \ldots, x_{1-1}, x_{1+1}, \ldots, x_{n+1}\right], 1<1<n+1
$$

have non-void intersection. Consequently the sets $k_{1}, 1<1<n+l$ have non-vold intersection. Now, suppose that the intersection of the members of each set of (card I) - 1 semi-sub-hypergroup's is non-void. So, for every $1 \varepsilon I$,, we plck an element $x_{1}$ which belongs to the intersection $\underset{j \neq 1}{n} k_{j}$. Then

$$
x_{1}=\left\{x_{j}, j \varepsilon I-\{i\}\right\} \subseteq K_{i}
$$

and therefore $\left[\mathrm{X}_{1}\right] \subseteq \mathrm{K}_{1}$. But every $n+1$ elements of $\Omega$ are correlated, thus the elements $\left\{x_{1}, 1 \varepsilon I\right\}$ are also correlated and because of Theorem 3.1 , the semi-subhypergroups $\left[\mathrm{X}_{1}\right], 1 \in I$ have non-void intersection. Consequently the sets $K_{1}$, $i \varepsilon I$ have non-void intersection.

In the case of the hypergroup $(V,$.$) , where V$ is a vector space, the correlated elements are directly connected with the affine dependance. It can be prove that the correlated elements are affinely dependent and that the affinely dependent elements are correlated. Indicatively let us show the first part of the above. Suppose that the elements $a_{1}, \ldots, a_{n}$ of $v$ are correlated. Then there are distinct integers $\lambda_{1}, \ldots, \lambda_{\nu}, k_{1}, \ldots, k_{\mu}$ such that

$$
\left[a_{\lambda 1}, \ldots, a_{\lambda \nu}\right] \cap\left[a_{K l}, \ldots, a_{K \mu}\right] \neq \phi \text {. }
$$


But because of the Theorem 2.1 the semi-sub-hypergroups of the form $\left[a_{\lambda 1}, \ldots, a_{\lambda \nu}\right]$ can be represented from the combinations of the monogene semi-sub-hypergroups [a ${ }_{\lambda}$ ]. So some intersections of these combinations are non-void. Also $\left[a_{1}\right]=a_{1}$. Thus, there are $\left\{i_{1}, \ldots, i_{k}\right\} \subseteq\left\{\lambda_{1}, \ldots, \lambda_{\nu}\right\}$ and $\left\{j_{1}, \ldots, j_{t}\right\} \subseteq\left\{k_{1}, \ldots, k_{\mu}\right\}$ such that

$$
a_{i 1} \cdot \cdots \cdot a_{i k} a_{j l} \cdot \cdots \cdot a_{j t} \neq \phi \cdot
$$

Now let $x$ be an element which belongs to this last intersection. Then

$$
\begin{aligned}
& x=r_{1} a_{i 1}+\ldots+r_{K} a_{1 k} \text { with } r_{1}+\ldots+r_{k}=1 \text { and } x=s_{1} a_{j 1}+\ldots+s_{t} a_{j t} \text { with } \\
& s_{1}+\ldots+s_{t}=1 \text {. Thus we have } \\
& \quad s_{1} a_{j 1}+\ldots+s_{t} a_{j t}+\left(-r_{1}\right) a_{11}+\ldots+\left(-r_{k}\right) a_{1 k}=0
\end{aligned}
$$

with $s_{1}+\ldots+s_{t}+\left(-r_{1}\right)+\ldots+\left(-r_{k}\right)=0$ but without all the coefficients s, $r$ being equal to 0 . Thus the elements $a_{1}, \ldots, a_{n}$ are affinely dependent.

After this remark we can take the Helly's Theorem (e.g. see Bronsted [14]) as a corollary of Theorem 3.2.

COROLlaRY 3.1. Let us consider a finite family $\left(C_{1}\right)_{1 \varepsilon I}$ of convex sets in $R^{d}$, with $d+1$ < card $I$. Then, if any $d+1$ of the sets $C_{1}$ have a non-empty intersection, all the sets $C_{1}$ have non-empty intersection.

\section{FUNDAMENTAL ELEMENTS.}

DEFINITION 4.1. Let $\alpha$ be an element of a semi-sub-hypergroup $H$ of a hypergroup $\Omega_{0} \quad$ Then $\alpha$ will be called fundamental element of $H$, if from the relation $\langle\alpha\rangle \cap x \cdot y \neq \phi$, it follows that $x, y \varepsilon\langle\alpha\rangle$, for every $x, y \varepsilon H$.

In what follows $\Omega$ will signify a hypergroup and $H$ a semi-sub-hypergroup of $\Omega$.

PROPOSITION 4.1. $\alpha$ is a fundamental element of $H$ if and only if $H-\langle\alpha\rangle$ is a semi-sub-hypergroup of $H$ or the void set.

PROOF. If $\alpha$ is a fundamental element and $x, y$ are in $H-\langle\alpha\rangle, x \cdot y$ is a subset of $H$ that does not intersect $\langle\alpha\rangle$. Hence $x \cdot y \subseteq H-\langle\alpha\rangle$ and so $H-\langle\alpha\rangle$ is a semtsub-hypergroup of $H$. Conversely, let $\alpha \varepsilon H$ and let $H-\langle\alpha\rangle$ be a semi-sub-hypergroup of $H$. Let us suppose that there are $x, y \in H$ such that

$$
\langle\alpha>n x \cdot y \neq \phi, \quad x, y \in H \text {. }
$$

Both $x, y$ can not belong to $H-\langle\alpha\rangle$, since $H-\langle\alpha\rangle$ is a semi-sub-hypergroup of $H$. If $x \varepsilon\langle\alpha\rangle$, then $\langle\alpha\rangle n\langle\alpha\rangle$. $y \neq \phi$, from which $y \varepsilon\langle\alpha\rangle$ : $\langle\alpha\rangle$ or equivalently y $\varepsilon\langle\alpha\rangle$. Therefore $\alpha 18$ fundamental. If $H-\langle\alpha\rangle=\emptyset$, the proof is trivial.

REMARK 4.1. Let $\alpha$ be an element of $H$. Then $\alpha 18$ a fundamental element of $H$ if and only if from the relation

$$
\left\langle\alpha>n \alpha_{1} \cdot \ldots \cdot \alpha_{n} \neq \phi, \quad\left\{\alpha_{1}, \ldots, \alpha_{n}\right\} \subseteq H, \quad n>2\right.
$$

we derive $\left\{\alpha_{1}, \ldots, \alpha_{n}\right\} \subseteq\langle\alpha\rangle$.

Indeed if $\langle\alpha\rangle$ intersects $\alpha_{1} \cdots \ldots \alpha_{n} \neq \phi$, then $\langle\alpha\rangle$ contains $\alpha_{1}$ and intersects $\alpha_{2}, \ldots, \alpha_{n}$ 
PROPOSITION 4.2. Suppose that there is a subset $A$ of $H$ such that $H=[A]$. Also let $\alpha$ be a fundamental element of $H$. Then

$$
\langle\alpha>\cap A \neq \phi \text {. }
$$

PROOF. Since $\mathrm{H}-\langle\alpha\rangle$ is a semi-sub-hypergroup or the vold set, if $A$ did not intersect $\langle\alpha\rangle$, it would at most generate $H-\langle\alpha\rangle$.

THEOREM 4.1. Let $H$ be commutative and $H=[A]$, for some subset $A$ of $H$. Also let $\alpha \varepsilon A$ be a non-fundamental element of $H$. Then

$$
\langle\alpha>\cap[A-\{a\}] \neq \phi \text {. }
$$

PROOF. From the assumption that $\alpha$ is a non-fundamental element, derives that there are $x, y \in H-\langle\alpha\rangle$ such that

$$
\langle\alpha\rangle n x \cdot y \neq \phi
$$

Since $H=[A]$ there are elements of $A$ such that

$$
x \in\left[\alpha_{1}\right] \cdot \ldots \cdot\left[\alpha_{n}\right]
$$

and

$$
\text { y } \varepsilon\left[\alpha_{n+1}\right] \cdot \ldots \cdot\left[\alpha_{k}\right]
$$

Now from the relations $(4.2)$ and $(4.3)$ we have

$$
x \cdot y \subseteq\left[\alpha_{1}\right] \cdot \ldots \cdot\left[\alpha_{n}\right] \cdot\left[\alpha_{n+1}\right] \cdot \ldots \cdot\left[\alpha_{k}\right]
$$

or eliminating the equal terms we get

$$
x \cdot y \subseteq\left[\beta_{1}\right] \cdot \ldots \cdot\left[\beta_{\mu}\right]
$$

Combining now (4.1) and (4.4) we have

$$
\langle\alpha\rangle \cap\left[\beta_{1}\right] \cdot \cdots \cdot\left[\beta_{\mu}\right] \neq \phi \cdot
$$

If in (4.5) holds it holds that $\left[\beta_{\ell}\right] \neq[\alpha]$, for every $\ell=1, \ldots, \mu$, then the theorem has already been proved, because

$$
\left[\beta_{1}\right] \cdot \cdots \cdot\left[\beta_{\mu}\right] \subseteq[A-\{\alpha\}]
$$

and

$$
\langle\alpha\rangle \cap[A-\{\alpha\} \neq \phi \text {. }
$$

But if we suppose that one of the $\left[\beta_{1}\right]$ 's say $\left[\beta_{1}\right]$, equals $[\alpha]$, then $\langle\alpha\rangle \cap[\alpha] \cdot\left[\beta_{2}\right] \cdot \ldots \cdot\left[\beta_{\mu}\right] \neq \phi . \quad$ From the last relation we derive that there are $v \varepsilon\langle a\rangle, w \varepsilon[\alpha]$ and $\beta \varepsilon\left[\beta_{2}\right] \ldots . . .\left[\beta_{\mu}\right]$ such that $v \varepsilon w_{0} \beta$, from which it follows that $\beta \varepsilon$ v: $\omega_{\text {. }}$ So $\beta \varepsilon\left\langle\alpha>\right.$. Hence $\beta \varepsilon<\alpha>\cap\left[\beta_{2}\right] \cdot \ldots \cdot\left[\beta_{\mu}\right]$. Thus $\left\langle\alpha>\cap\left[\beta_{2}\right] \cdot \ldots \cdot\left[\beta_{\mu}\right] \neq \phi\right.$ and this completes the proof.

According to Proposition 2.8, the set $\left[\beta_{1}\right] . \ldots .\left[\beta_{\mu}\right]$ of the relation (4.5) of the above theorem, is a semi-sub-hypergroup of $H$. So if

$x \in[\alpha] \cap\left[\beta_{1}\right] \cdot \ldots \cdot\left[\beta_{1}\right] \cdot \ldots \cdot\left[\beta_{\mu}\right]$, then

$$
[\mathbf{x}] \subseteq[a] \quad\left[\beta_{1}\right] \cdot \cdots \cdot\left[\beta_{\mu}\right]
$$

Now, assume that the sets $[x], x$ in $H$, are a partition of $H$. Then from (4.6) we get 
tha: $[x]=[\alpha]$. Thus the relation $[\alpha \mid[A-\{\alpha\}] \neq \phi$ becomes $[\alpha] \subseteq[A-\{\alpha\}]$ and so we have ihe Curollary.

COROLLARY 4.1. Let $H$ be commutalive and $H=[A]$, for some subset $A$ of $H$. Suppose that the sets $[x], x$ in $H$, are a particion of $H$. Then $[A-\{\alpha\}]=H$, if $\alpha$ is a nonfundamental element.

Thus we observe that there are cerlain elements which always participate in any set of generators, either by ihemselves, or through those elements that derive from thelr monogene sub-hypergroups. Even though these elements are fundamenial (and this is why we have named them so) for the genesis of the semi-sub-hypergroup, there are times that they can not produce the semi-sub-hypergroup by themselves. On the other hand we can define the nongenerators of a semi-sub-hypergroup $H$ in the same way, as they are defined in the classical case in group theory $1 . e . t$ is called a nongenerator of $H$ if $H=[t, X]$ always implies that $H=[X]$, when $X$ is a subset of $H$. So the nongenerators are the concept antipodal of the fundamental elements and when their set is non-void, they form a semi-sub-hypergroup $\phi(H)$ of $H$ whlch we shall call Frattini's semi-sub-hypergroup. If we would like to have an optical view of the fundamental elemenis and Frattin's semi-sub-hypergroup we could consider the vector space $R^{2}$ in example (v). In this hypergroup any convex angle is a semi-sub-hypergroup. The only fundamental element of a convex angle is its vertex, while all the other points (elments) of the angle are nongenerators and they form the Frattini's semi-subhypergroup. Of course it is obvious that the only fundamental element of the angle can not generate the angle by itself.

\section{REFERENCES}

1. MARTY, F. Sur in généralisation de la notion de group. Huitieme Congres des Matimaticiens Scad; (Stockholm 1934, 45-59).

2. MITTAS, J. Hypergroupes Canoniques. Mathematlca Balkanica t. 2, (1972), 165-179.

3. MITTAS, J Sur une classe d' Hypergroupes Commutatifs C.R. Acad. Sc. (Paris) t. 269, (1969), 485-488, Serie A.

4. KRASNER, M. Une Nouvelle Présentation de la Théorie des Groupes de Permutations et ses Applifations a' la Theorle de Galois et de Produit d' Entrelacement ("Wreath Product") de groupes. Math. Balk. t.3, (1973), 229280 .

5. ROTH, R.L. Character and Conjugacy Class Hypergroups of a Finite Group. Ann. Math. Pura Appl. 105 (1975), 295-311.

6. KRASNER, M. Approximation des Corps Values Complets de Caractéristique $p \neq 0$ par ceux de Caractéristique 0. Colloque d' Algebre Superieure (Bruxelles, December 1956), CBRM, Bruxelles, 1957.

7. MASSOUROS, $\mathrm{CH} . \mathrm{G}$. On the Theory of Hyperrings and Hyperfields. Algebra 1 Logika 24, NO. $6,728-742,1985$.

8. NAKASSIS, A. Recent Results in Hyperring and Hyperfield Theory, Internat. J. Math.\& Math. Sci., 1987. 
9. MASSOUROS, CH.G. Free and Cyclic Hypermodules. Annali Di Mathematica Pura ed Applicata. Tomo CL, (1988), 153-166.

10. MITTAS, J. Espaces Vectoriels sur un Hypercorps - Introduction des Hyperspaces affines et Euclidiens. Mathematica Balkanica t. 5, (1975), 199-211.

11. MASSOUROS, $\mathrm{CH}$. G. Methods of Constructing Hyperfields, Internat. J. Math. \& Math. Sc1. 8(4), (1985), 725-728.

12. VOUGIOUKLIS, T. Cyclicity of Hypergroups, Doctoral Thesis, Democritus University of Thrace, Greece 1980.

13. VOUGIOUKLIS, T. Cyclicity in a Special Class of Hypergroups, Acta Universitatis Carolinae-Mathematica et Physica. 22(1) (1981).

14. BRONSTED, A. An Introduction to Convex Polytopes, Springer-Verlag, 1983.

15. PRENOWITZ, W. and JANTOSCIAK, J. Geometries and Join Spaces, J. Reine Angew. Math. 257 (1972), 100-128. 


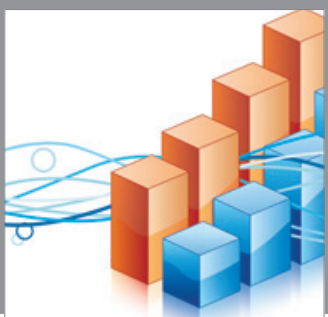

Advances in

Operations Research

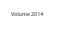

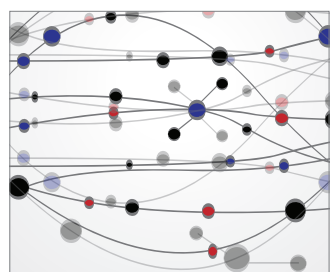

\section{The Scientific} World Journal
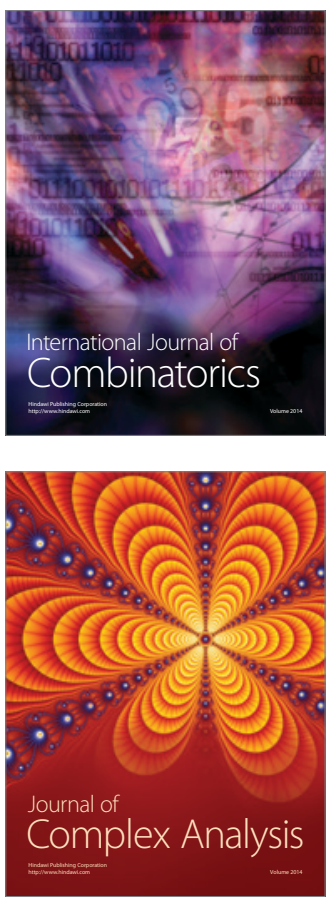

International Journal of

Mathematics and

Mathematical

Sciences
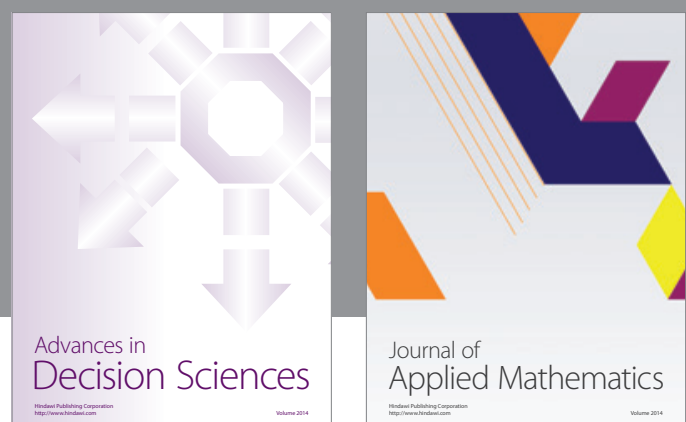

Journal of

Applied Mathematics
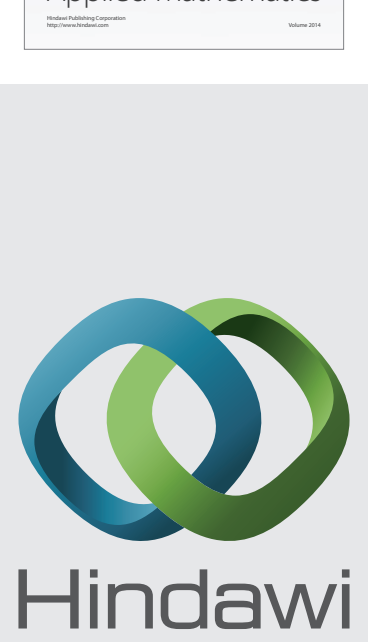

Submit your manuscripts at http://www.hindawi.com
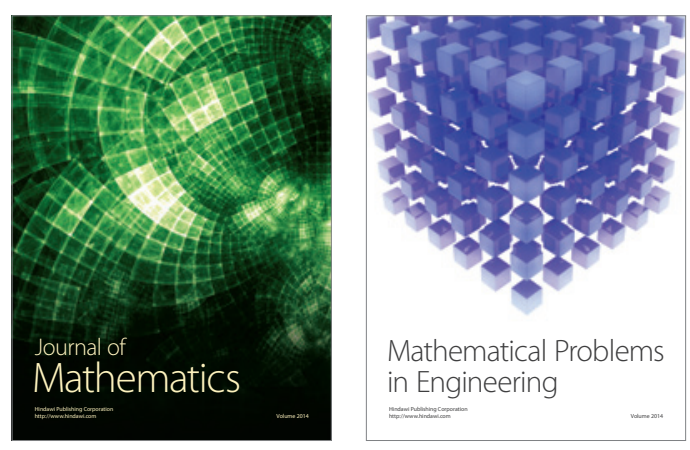

Mathematical Problems in Engineering
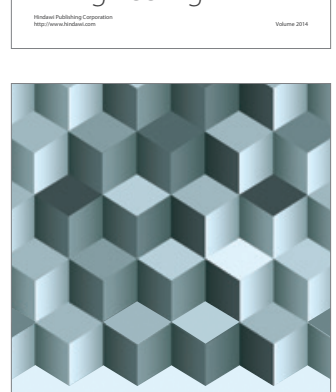

Journal of

Function Spaces
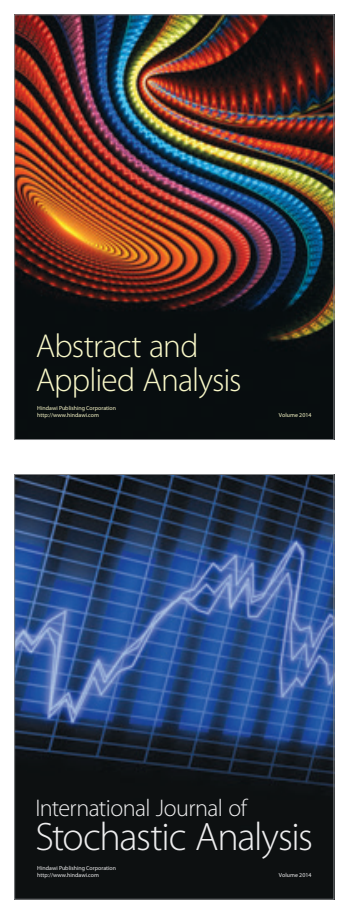

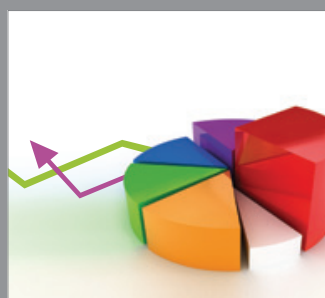

ournal of

Probability and Statistics

Promensencen
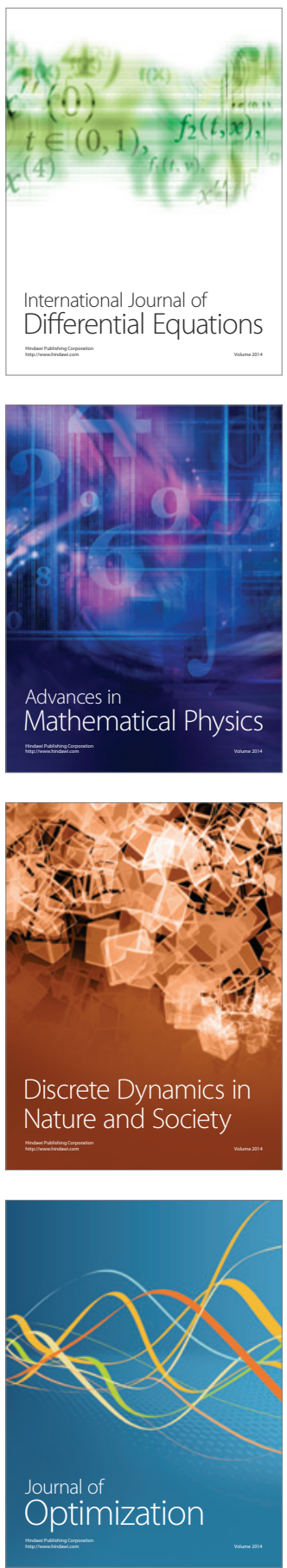